\title{
Ectopic expression of Arabidopsis L-type lectin receptor kinase genes LecRK-I.9 and LecRK-IX.1 in Nicotiana benthamiana confers Phytophthora resistance
}

\author{
Yan Wang ${ }^{1,2} \cdot$ David L. Nsibo ${ }^{1} \cdot$ Hagos M. Juhar ${ }^{1} \cdot$ Francine Govers $^{1}$ • \\ Klaas Bouwmeester ${ }^{1,3}$
}

Received: 14 July 2015/Revised: 8 December 2015/ Accepted: 29 December 2015/Published online: 21 January 2016

(C) The Author(s) 2016. This article is published with open access at Springerlink.com

\begin{abstract}
Key message Transgenic Nicotiana benthamiana lines with constitutive expression of an Arabidopsis lectin receptor kinase gene (LecRK-I.9 or LecRK-IX.1) show enhanced resistance to Phytophthora pathogens, demonstrating conserved gene functionality after interfamily transfer.

Abstract In plants, cell surface receptors mediate the first layer of innate immunity against pathogenic microbes. In Arabidopsis several L-type lectin receptor kinases (LecRKs) were previously found to function as Phytophthora resistance components. In this study, we determined the functionality of Arabidopsis LecRK-I.9 or LecRK-IX.1 in Phytophthora resistance when transferred into the Solanaceous plant Nicotiana benthamiana. Multiple transgenic lines were generated for each LecRK gene and molecular analyses revealed variation in transgene copy number, transgene expression levels and LecRK protein accumulation. Infection assays showed that transgenic $N$. benthamiana plants expressing either Arabidopsis LecRK-I.9 or LecRK-IX.1 are more resistant to
\end{abstract}

Communicated by H. S. Judelson.

F. Govers and K. Bouwmeester have contributed equally to this work.

Klaas Bouwmeester

klaas.bouwmeester@wur.nl; K.Bouwmeester@uu.nl

1 Laboratory of Phytopathology, Plant Sciences Group, Wageningen University, Wageningen, The Netherlands

2 Department of Plant Pathology, Nanjing Agricultural University, Nanjing, People's Republic of China

3 Plant-Microbe Interactions, Department of Biology, Faculty of Science, Utrecht University, Utrecht, The Netherlands
Phytophthora capsici and to Phytophthora infestans. These results demonstrate that Arabidopsis LecRK-I.9 and LecRK-IX.1 retained their Phytophthora resistance function when transferred into $N$. benthamiana. Therefore, these LecRKs have the potential to function as a complementary Phytophthora resistance resource in distantly related plant species next to the canonical Phytophthora resistance genes encoding nucleotide-binding leucine-rich repeat proteins.

Keywords L-type lectin receptor kinases · LecRK . Phytophthora $\cdot$ Disease resistance .

Nicotiana benthamiana - Interfamily gene transfer

\section{Introduction}

Plant diseases caused by Phytophthora species are widespread and cause enormous economic losses on a large variety of crops (Tyler 2007; Fry 2008; Lamour et al. 2012). Under favorable conditions, Phytophthora pathogens reproduce rapidly and become epidemic within a short period of time. Phytophthora disease control is costly and often depends on excessive application of preventive fungicides. Hence, development of plant cultivars with durable resistance against different Phytophthora species is under high demand. Currently, breeding programs are mainly focused on the exploitation of resistance $(R)$ genes that encode intracellular nucleotide-binding leucine-rich repeat (NLR) proteins to restrict Phytophthora pathogens (Vleeshouwers et al. 2011). However, these attempts are often hampered by the quick adaptation of Phytophthora pathogens that leads to evasion of the $R$-gene mediated recognition (Fry 2008; Vleeshouwers et al. 2011; Rodewald and Trognitz 2013). 
Plants also respond to pathogens by activation of plasma membrane-localized receptor-like kinases (RLKs) that function as pattern recognition receptors (PRRs) to initiate defense (Zipfel 2014). Plant resistance mediated by PRRs has been hypothesized to confer broad-spectrum resistance against plant pathogens, but thus far received little attention in resistance breeding of crop plants. One of the largest classes of RLKs comprising potential PRRs are the L-type lectin receptor kinases (LecRKs). LecRKs are ubiquitously present throughout the plant kingdom. Arabidopsis has 45 LecRK genes, several of which belong to evolutionary conserved clades whereas others are species- or genusspecific (Bouwmeester and Govers 2009; Hofberger et al. 2015; Wang et al. 2015a).

Thus far, several LecRKs have been found to play a role in plant resistance to different Phytophthora pathogens. Arabidopsis LecRK-I.9 was the first one described to function as a Phytophthora resistance component (Bouwmeester et al. 2011). To unravel the function of other Arabidopsis LecRKs, a large set of T-DNA insertion mutants covering 36 of the 45 LecRKs was analysed (Wang et al. 2014) and infection assays revealed that mutants of 13 LecRKs showed compromised Phytophthora resistance. These included mutants of the previously identified LecRK-I.9 and of the two members of clade IX, namely LecRK-IX.1 and LecRK-IX-2. More recently, the latter two were analysed in more detail and this confirmed that they both function as Phytophthora resistance component in Arabidopsis (Wang et al. 2015b).

Engineering plants via interfamily transfer of resistance components has the potential to improve disease resistance in crop plants. A successful example is the transfer of Arabidopsis EFR into Solanaceous plants (Lacombe et al. 2010). EFR is a receptor of bacterial elongation factor EFTU and is restricted to the Brassicaceae family (Kunze et al. 2004; Zipfel et al. 2006). Nicotiana benthamiana and tomato transgenic plants expressing EFR gained the capacity to respond to EF-Tu and showed enhanced resistance to various bacterial pathogens (Lacombe et al. 2010). In a similar way, Arabidopsis LecRK-I.9 as transgene in potato was shown to confer enhanced resistance to Phytophthora infestans (Bouwmeester et al. 2014). Consistently, transient expression of LecRK-I.9 in $N$. benthamiana also resulted in increased resistance to Phytophthora pathogens (Bouwmeester et al. 2014) and the same holds for LecRK-IX.1 and LecRK-IX.2 (Wang et al. 2015b). Likewise, Arabidopsis LecRK-VI.2 maintained its function in bacterial resistance when expressed in $N$. benthamiana (Huang et al. 2014).

The objective of this work was to check whether the Arabidopsis lectin receptor kinase genes LecRK-I.9 and
LecRK-IX.1 maintain their functionality in Phytophthora resistance when stably integrated as transgene in the distantly related species $N$. benthamiana. To this end, transgenic N. benthamiana plants ectopically expressing either Arabidopsis LecRK-I.9 or LecRK-IX.I were generated using Agrobacterium-mediated transformation. The obtained transgenic lines were subjected to molecular analyses to determine transgene copy number, transgene expression level and LecRK protein accumulation. Thereafter, we monitored the phenotypic changes of these transgenic $N$. benthamiana lines in growth and response to different Phytophthora pathogens. Since $N$. benthamiana is a model plant amenable for virus-induced gene silencing and is widely used for studying plant-pathogen interactions and protein-protein interactions, these transgenic plants are valuable as experimental tool for further functional analysis of LecRKs.

\section{Materials and methods}

\section{Plant growth conditions and infection assays}

Nicotiana benthamiana seeds were surface-sterilized by $70 \%$ ethanol and $1 \% \mathrm{NaClO}$, and grown on MS medium (4.4 g MS salt, $20 \mathrm{~g}$ sucrose and $8 \mathrm{~g}$ agar) or MS medium supplemented with antibiotics in a conditioned growth chamber at $19-21{ }^{\circ} \mathrm{C}$, with a $16 \mathrm{~h}$ photoperiod and a relative humidity of $75-80 \%$. Plants grown in soil were kept in a greenhouse with similar settings. Supplementary light $\left(100 \mathrm{~W} \mathrm{~m}^{-2}\right)$ was applied when the light intensity dropped below $150 \mathrm{~W} \mathrm{~m}^{-2}$.

Phytophthora capsici isolates LT263 and LT3239 were maintained in the dark on V8 plates at $25^{\circ} \mathrm{C}$ (Wang et al. 2013), and P. infestans isolate 14-3-GFP on rye sucrose agar at $18{ }^{\circ} \mathrm{C}$ (Bouwmeester et al. 2014). $P$. infestans zoospores were obtained by treating sporulating mycelia with cold water for 3-4 h. For detachedleaf assays, leaves from 5-week-old $N$. benthamiana plants were collected and the abaxial sides were inoculated with $P$. capsici mycelial plugs $(\varnothing 0.5 \mathrm{~cm})$ or $10 \mu \mathrm{L}$ droplets of a $P$. infestans zoospore suspension with a concentration of $5 \times 10^{5}$ zoospores $\mathrm{mL}^{-1}$. Inoculated leaves were kept in transparent plastic boxes with high humidity in the dark overnight and thereafter exposed to a condition with a $12 \mathrm{~h}$ photoperiod and appropriate temperature settings. Disease severity was monitored by measuring lesion sizes (Vleeshouwers et al. 1999) 3 and 6 days after inoculation with $P$. capsici and $P$. infestans, respectively. 


\section{Agrobacterium-mediated transformation} of $N$. benthamiana

A. tumefaciens GV3101 carrying the binary vectors pBINKS-35S::AtLecRK-I.9-eGFP, pBIN-KS-35S::AtLecRKIX.1-eGFP (Wang et al. 2015b) and pBIN61-35S::GFP (Fig. 2a) were grown overnight at $28{ }^{\circ} \mathrm{C}$ in Yeast Extract Broth with appropriate antibiotics. A. tumefaciens cells were pelleted, resuspended and incubated in MMA induction medium (10 mM MES, $10 \mathrm{mM} \mathrm{MgCl}_{2}, 50 \mu \mathrm{M}$ acetosyringone, $\mathrm{pH}$ 5.6) for $3 \mathrm{~h}$. A. tumefaciens cells were collected by centrifugation and resuspended in MS broth supplemented with $150 \mu \mathrm{M}$ acetosyringone. Leaf pieces $\left(2-3 \mathrm{~cm}^{2}\right)$ were cut from 5-week-old $N$. benthamiana leaves and incubated with A. tumefaciens cells for $30 \mathrm{~min}$. Thereafter, leaf discs were dried on filter paper to remove excess A. tumefaciens and incubated on regeneration medium consisting of MS salt, $1 \mathrm{mg} / \mathrm{L}$ cytokinins 6-BAP (6-benzyl amino purine), $0.1 \mathrm{mg} / \mathrm{L}$ auxin NAA (1-naphthaleneacetic acid) and $0.8 \%$ agar for 2 days at $19-21{ }^{\circ} \mathrm{C}$. Leaf pieces were transferred every week to fresh regeneration medium supplemented with $400 \mathrm{mg} / \mathrm{L}$ cefotaxime, $200 \mathrm{mg} / \mathrm{L}$ vancomycin and $200 \mathrm{mg} / \mathrm{L}$ kanamycin until the appearance of plantlets. The generated plantlets were transferred to MS medium containing $200 \mathrm{mg} / \mathrm{L}$ kanamycin to allow root development. Upon root generation, plantlets were transferred into soil and kept in the greenhouse for seed harvesting.

\section{Transgene detection in transgenic $N$. benthamiana}

Genomic DNA was isolated using CTAB buffer $(0.02 \%$ CTAB, 100 mM Tris-HCl pH 8.0, 20 mM EDTA pH 8.0, $1.4 \mathrm{M} \mathrm{NaCl}$ and $1 \% \mathrm{PVP}$ ) followed by precipitation with isopropanol. RNA was removed by RNaseA. Transgene presence was checked by PCR using specific primers for AtLecRK-I.9 and AtLecRK-IX.I and a primer matching a flanking sequence in the binary vector (Table 1; Fig. 2a).

Transgene copy number in $\mathrm{T}_{0}$ transgenic lines was determined according to Honda et al. (2002). Briefly, qPCR was performed using genomic DNA as template with specific primers for AtLecRK-I.9, AtLecRK-IX.1, the neomycin phosphotransferase II gene (NPTII) or NbActin (Table 1). The copy number was calculated by normalizing the amplification of AtLecRK-I.9, AtLecRK-IX.1 or NPTII to NbActin.

\section{RNA isolation and qRT-PCR}

Total RNA was isolated from leaves collected from 6-week-old $\mathrm{T}_{0}$ transgenic $N$. benthamiana plants with a NucleoSpin RNA plant Kit (Macherey-Nagel) and thereafter used as template for cDNA synthesis with an oligo-dT primer and a M-MLV reverse transcriptase kit (Promega). qRT-PCR was performed as previously described (Wang et al. 2014). Transcript levels of AtLecRK-I.9 and AtLecRK-IX.1 were normalized to NbActin.

\section{Protein extraction, immunoprecipitation and western blotting}

Leaves collected from 6-week-old $\mathrm{T}_{0}$ transgenic $N$. benthamiana plants were ground in liquid nitrogen and subsequently incubated for $30 \mathrm{~min}$ in an extraction buffer containing $150 \mathrm{mM} \mathrm{NaCl}, 50 \mathrm{mM}$ Tris- $\mathrm{HCl} \mathrm{pH} 8.0,1.0 \%$ IGEPAL $^{\circledR}$ CA-630 (Sigma) and one protease inhibitor cocktail tablet per $50 \mathrm{~mL}$ (Roche). The homogenate was centrifuged at $18,000 \mathrm{rpm}$ for $20 \mathrm{~min}$ and the supernatant

Table 1 Primers used in this study

\begin{tabular}{lll}
\hline Primer & & \multicolumn{1}{c}{ Sequence $5^{\prime}-3^{\prime}$} \\
\hline NPTII-RT-F & GGAGAGGCTATTCGGCTATG & Used to/for \\
NPTII-RT-R & TCGTCCTGCAGTTCATTCAG & Check presence of $N P T I I$ \\
Nbactin-F & TATGGAAACATTGTGCTCAGTGG & Check presence of NPTII \\
Nbactin-R & CCAGATTCGTCATACTCTGCC & Endogenous control \\
Oligo-dT & GACTCGAGTCGACATCGATTTTTTTTTTTTTTT & Endogenous control \\
pGRAB-F1 & CCCACTATCCTTCGCAAGACCCTTCC & cDNA synthesis \\
IX.1-RT-F & CAAGGCGAGTAATGTGATGCT & Check presence of T-DNA \\
IX.1-RT-R & TAACCAAATGTTCCTGCTAACC & Check presence of AtLecRK-IX.1; qRT-PCR \\
IX.1-F & TCAAGCCTGGAGCTAATAG & qRT-PCR \\
IX.1-R & ACGACCATGTTGAGCACTTG & Check presence of AtLecRK-IX.1 \\
I.9-RT-F & TTTGCCAGATTTCTCACCATACAC & Check presence of AtLecRK-IX.1 \\
I.9-RT-R & TCTGTTGACTGCCAAGCGTAAG & qRT-PCR \\
I.9-F & ATGGCTCGTTGGTTGCTTCAG & qRT-PCR \\
I.9-R & GCTTTGACATCTCGGTGCAGAAC & Check presence of AtLecRK-I.9 \\
\hline
\end{tabular}


Table 2 Transgenic $N$. benthamiana lines vary in transgene copy number, gene expression and growth

\begin{tabular}{|c|c|c|c|c|c|c|}
\hline \multirow[t]{2}{*}{$\begin{array}{l}\text { Line } \\
\text { no. }\end{array}$} & \multicolumn{2}{|c|}{ Transgene copy number } & \multicolumn{2}{|c|}{$\begin{array}{l}\text { Relative transgene } \\
\text { expression }^{\mathrm{a}}\end{array}$} & \multirow[t]{2}{*}{ Morphology $\left(\mathrm{T}_{0} \text { transformants vs. wild type }\right)^{\mathrm{b}}$} & \multirow[t]{2}{*}{ Germination rate $^{c}$} \\
\hline & AtLecRK-I.9/IX.1 & NPTII & AtLecRK-I.9 & AtLecRK-IX.1 & & \\
\hline \multicolumn{7}{|l|}{$E V-$} \\
\hline 1 & 0 & 1 & n.d. & n.d. & - & 100 \\
\hline 2 & 0 & 2 & n.d. & n.d. & $-{ }^{\mathrm{e}}$ & 100 \\
\hline 3 & 0 & 5 & n.d. & n.d. & - & 100 \\
\hline 4 & 0 & 2 & n.d. & n.d. & $-{ }^{\mathrm{e}}$ & 100 \\
\hline \multicolumn{7}{|c|}{ I.9-OE- } \\
\hline 1 & 1 & 1 & 5.0 & & Slightly smaller & 32 \\
\hline 2 & 2 & 2 & 5.6 & & Smaller plants with curly leaves & 60 \\
\hline 3 & 3 & 3 & 6.2 & & Smaller plants with compacted rosette ${ }^{\mathrm{e}}$ & 40 \\
\hline 4 & 3 & 3 & 1.0 & & - & 60 \\
\hline 5 & 1 & 1 & 3.7 & & $-{ }^{\mathrm{e}}$ & 100 \\
\hline 6 & 4 & 4 & 7.8 & & - & 55 \\
\hline 7 & 0 & 1 & n.d. & & $-^{\mathrm{e}}$ & $f^{\mathrm{d}}$ \\
\hline 8 & 1 & 1 & 11.1 & & - & 60 \\
\hline 9 & 1 & 1 & 2.8 & & Smaller & 85 \\
\hline 10 & 5 & 6 & 59.4 & & Smaller & 95 \\
\hline 11 & 4 & 6 & 21.7 & & Smaller $^{\mathrm{e}}$ & 65 \\
\hline 12 & 1 & 1 & 4.1 & & $\begin{array}{l}\text { Smaller plants with compacted rosette, thick } \\
\text { leaves }\end{array}$ & 55 \\
\hline 13 & 2 & 2 & 3.9 & & - & 50 \\
\hline 14 & 2 & 2 & 3.6 & & - & 70 \\
\hline 15 & 1 & 1 & 2.3 & & Smaller $^{\mathrm{e}}$ & 60 \\
\hline 16 & 1 & 1 & 81.9 & & Smaller plants with compacted rosette & 35 \\
\hline 17 & 3 & 3 & 47.3 & & Slightly smaller and curly leaves & 85 \\
\hline \multicolumn{7}{|c|}{$I X .1-O E-$} \\
\hline 1 & 2 & 2 & & 1.7 & $-{ }^{\mathrm{e}}$ & 100 \\
\hline 2 & 1 & 2 & & 3.7 & Smaller plants with curly round leaves ${ }^{\mathrm{e}}$ & 100 \\
\hline 3 & 1 & 2 & & 9.0 & Smaller & 100 \\
\hline 4 & 2 & 2 & & 2.0 & $-{ }^{\mathrm{e}}$ & 100 \\
\hline 5 & 2 & 3 & & 2.0 & $-{ }^{\mathrm{e}}$ & 100 \\
\hline 6 & 1 & 2 & & 2.4 & - & 100 \\
\hline 7 & 1 & 1 & & 11.2 & Smaller ${ }^{\mathrm{e}}$ & 100 \\
\hline 8 & 2 & 2 & & 1.0 & - & 100 \\
\hline 9 & 1 & 1 & & 3.4 & - & 100 \\
\hline 10 & 1 & 1 & & 63.2 & Smaller plants with old leaves showing necrosis ${ }^{\mathrm{e}}$ & 100 \\
\hline 11 & 1 & 1 & & 87.3 & Smaller plants with old leaves showing necrosis & 100 \\
\hline 12 & 1 & 1 & & 90.3 & Smaller plants with old leaves showing necrosis ${ }^{\mathrm{e}}$ & 100 \\
\hline
\end{tabular}

a n.d. not detectable

b - , no difference compared with wild-type $N$. benthamiana

c Percentage of germinated seeds of $\mathrm{T}_{1}$ progeny lines $(n=18-24)$ after 3 days on MS

d / not tested

e Similar morphology in $\mathrm{T}_{1}$ progeny lines harboring the transgene

was incubated with GFP-trap_A ${ }^{\circledR}$ beads (Chromotek) at $4{ }^{\circ} \mathrm{C}$ for $1-2 \mathrm{~h}$. After incubation, GFP-beads were spinned down and washed six times with extraction buffer. Proteins were eluted from GFP-trap_A ${ }^{\circledR}$ beads by boiling for $5 \mathrm{~min}$, separated on an $8 \%$ SDS-PAGE gel and electroblotted onto PVDF membrane (Bio-Rad). Accumulation of eGFP- 


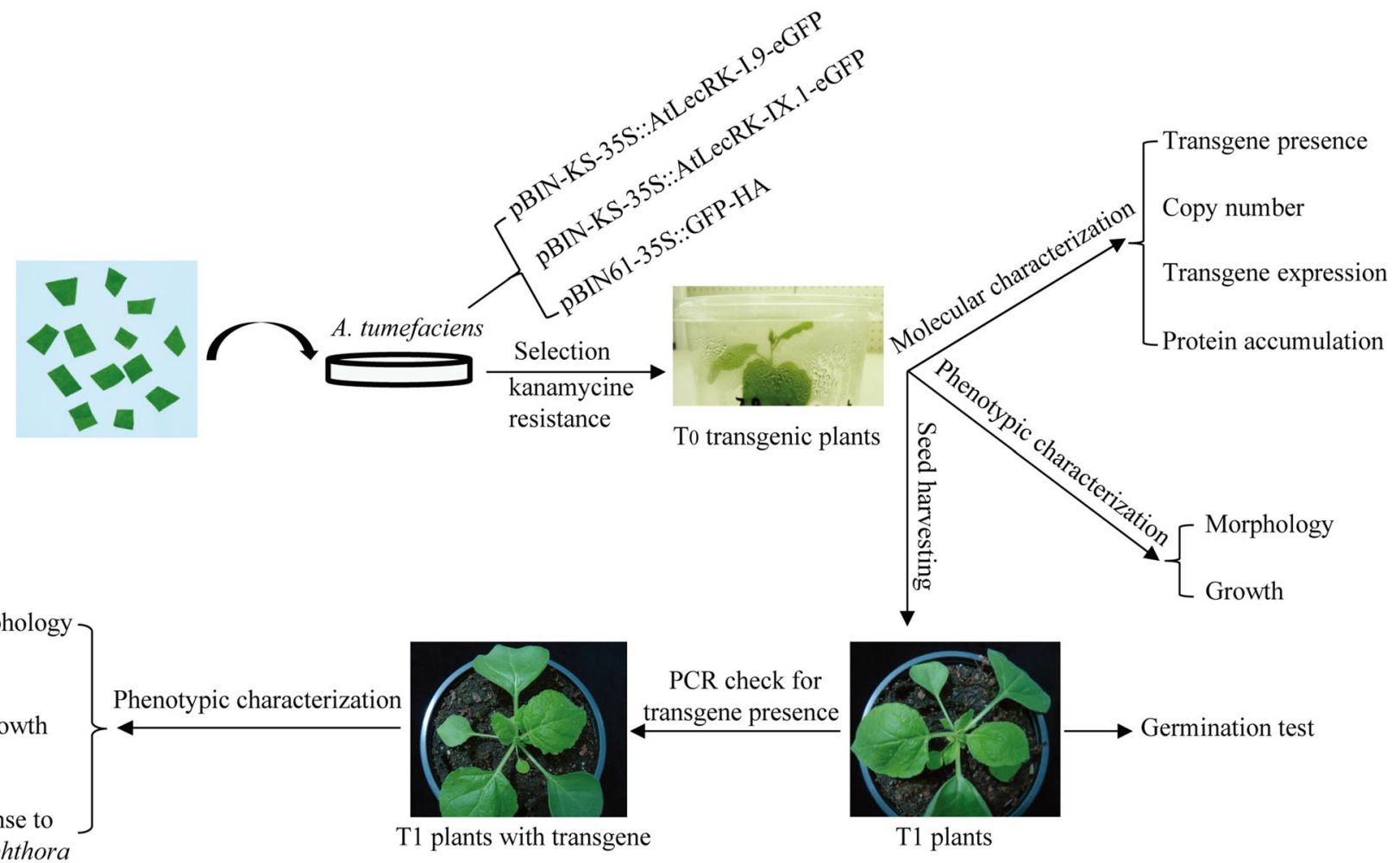

Fig. 1 Flowchart of the generation, selection and characterization of transgenic N. benthamiana lines harboring Arabidopsis LecRK-I.9 or LecRK-IX.1

tagged LecRK-I.9 and LecRK-IX.1 was detected by immunoblotting using anti-GFP-HRP (Miltenyi Biotec). Supersignal West Femto Chemiluminescent Substrate (Thermo Scientific) was used for signal development. Coomassie brilliant blue staining was used to indicate the amount of loading.

\section{Results and discussion}

\section{Generation of transgenic $N$. benthamiana ectopically expressing Arabidopsis LecRK-I.9 or LecRK-IX.1}

Agrobacterium-mediated transformation of $N$. benthamiana with the binary vectors pBIN-KS-35S::AtLecRK-I.9eGFP, pBIN-KS-35S::AtLecRK-IX.1-eGFP and pBIN6135S::GFP (Fig. 2a) resulted in 17, 12 and $4 \mathrm{~T}_{0}$ kanamycinresistant lines, respectively. These were named as I.9-OE1-17, IX.1-OE-1-12 and EV-1-4 (Table 2). The flowchart in Fig. 1 gives an overview of the various steps in the process of selection and analysis of the $T_{0}$ and $T_{1}$ transgenic lines. For the molecular characterization, we first determined whether AtLecRK-I.9 and AtLecRK-IX.1 were successfully transferred into $N$. benthamiana by PCR using gene-specific primers (Fig. 2a). Of the selected kanamycin-resistant plants, the four empty vector transgenic lines (EV-1 to EV-4) and one LecRK-I.9 line, i.e. I.9-OE-7, gave no PCR product (Fig. 2b).

To determine transgene copy number, we performed qPCR analysis. For both AtLecRK-I.9 and AtLecRK-IX.1, the copy number in transgenic lines ranged from 0 to 5 and this number was not always consistent with that of the determined NPTII copy number (Table 2). In the transgenic lines I.9-OE-7, I.9-OE-10, I.9-OE-11, IX.1-OE-2, IX.1-OE-3, IX.1-OE-5 and IX.1-OE-6, more copies were detected for NPTII than for LecRKs, indicating the presence of truncated T-DNA fragments. This likely explains why the $\mathrm{T}_{0}$ line I.9-OE-7 lacks AtLecRK-I.9 but is still kanamycin-resistant.

Expression of AtLecRK-I.9 and AtLecRK-IX.1 in the $\mathrm{T}_{0}$ plants was determined by quantifying mRNA levels using qRT-PCR. For both AtLecRK-I.9 and AtLecRK-IX.1, the expression level varied among individual transgenic lines (Fig. 2c). Variations in transgene expression level in stable transgenic lines have often been attributed to the site(s) of transgene insertion and transgene copy number (Kole et al. 2010). In $\mathrm{T}_{0}$ plants, however, no correlation was found between copy number and transgene expression level. Transgene expression level varied among individual transgenic lines with the same transgene copy number. Some of the transgenic lines with a single copy of the transgene showed even higher expression levels than those 
pBIN-KS-35S::AtLecRK-I.9-eGFP

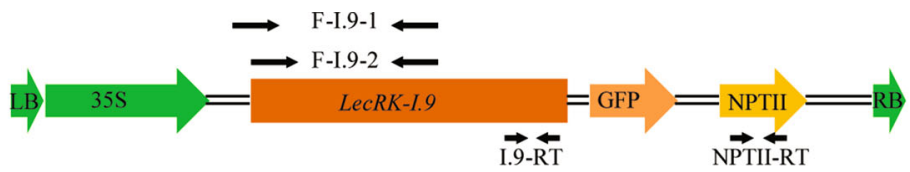

pBIN-KS-35S::AtLecRK-IX.1-eGFP

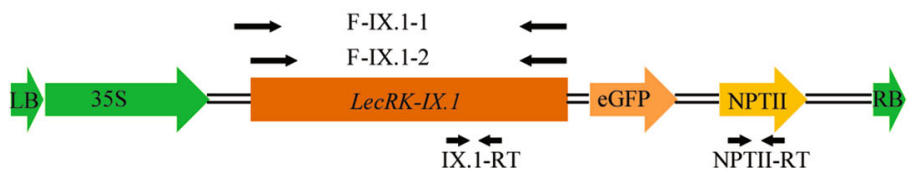

pBIN61-35s::GFP

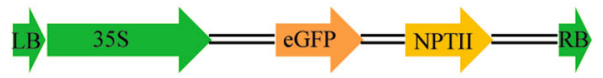

b
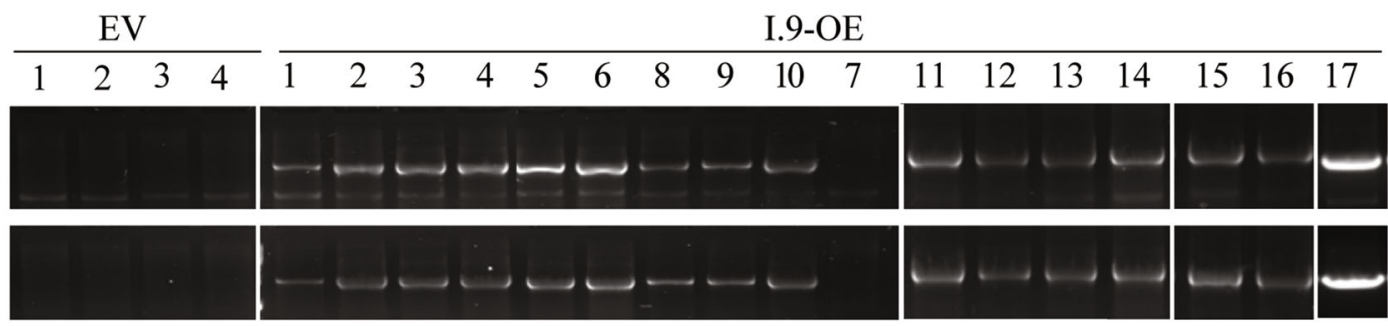

F-I.9-2

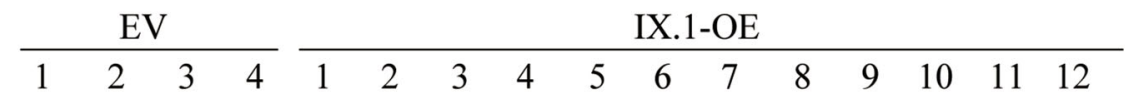

F-IX.1-1

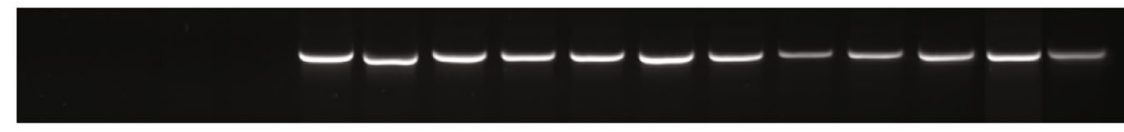

F-IX.1-2

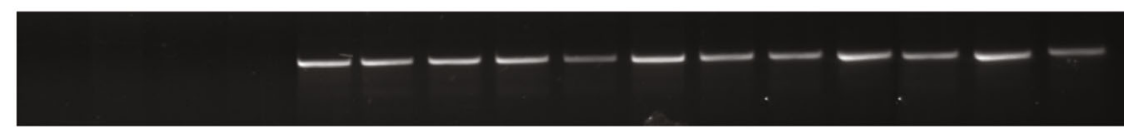

c

AtLecRK-I. 9

AtLecRK-IX.1
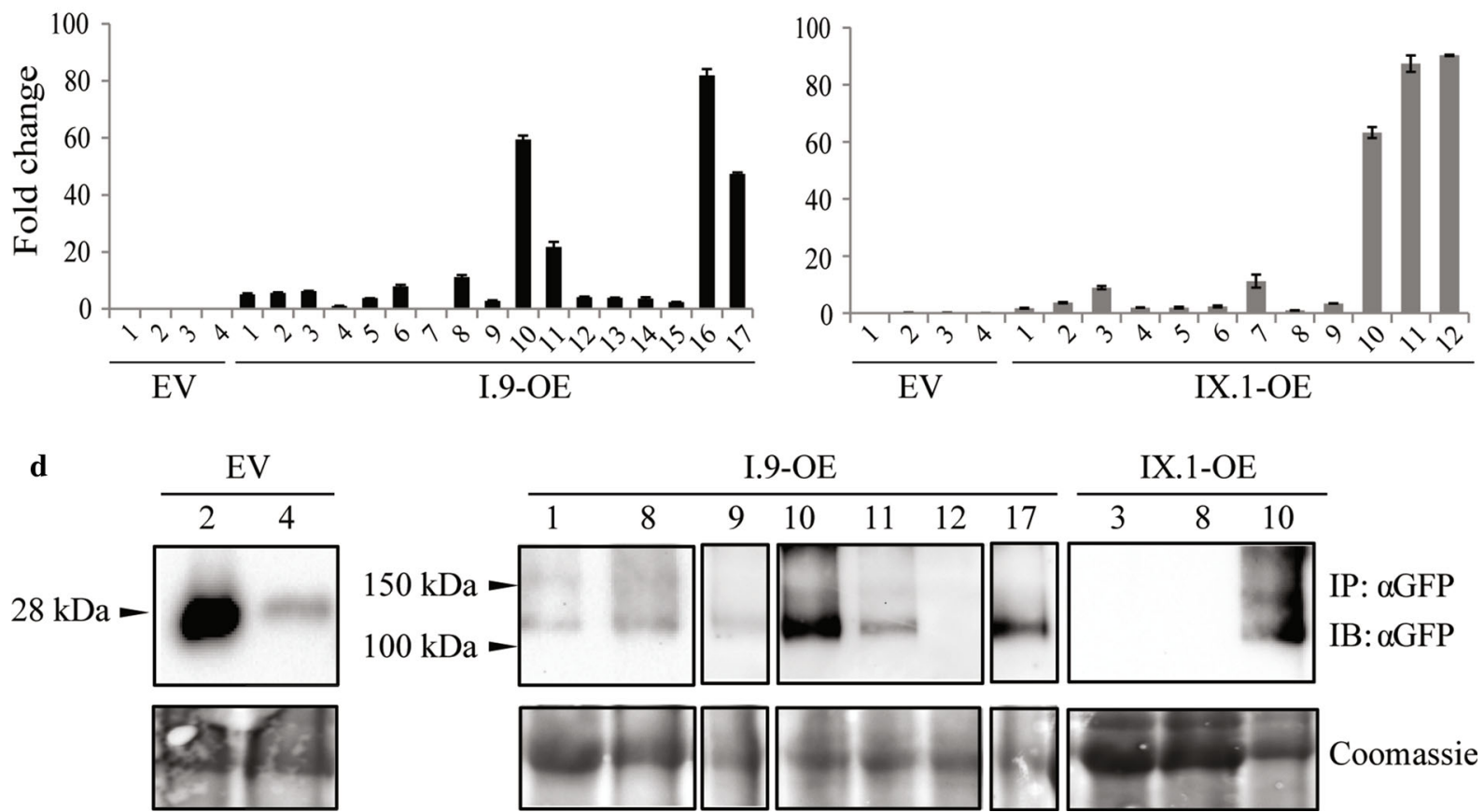

IP: $\alpha$ GFP

IB: $\alpha \mathrm{GFP}$

Coomassie 
४Fig. 2 Molecular characterization of transgenic $N$. benthamiana lines. a Schematic representation of T-DNA regions of the vectors used for $N$. benthamiana transformation. PCR amplified fragments and position of the primers are indicated by the head-to-head arrow pairs. The fragments F-I.9-1, F-I.9-2, F-IX.1-1, F-IX.1-2 and NPTII$R T$ were amplified to determine transgene presence in transgenic lines, while fragments $I .9-R T$ and $I X .1-R T$ were amplified to monitor transgene mRNA levels. b Presence of AtLecRK-I.9 or AtLecRK-IX.1 in transgenic $N$. benthamiana lines. Genomic DNA from each line was used as template for amplification with primers indicated in (a). c Relative quantification of transgene expression levels in transgenic $N$. benthamiana lines. Transcript levels were normalized to NbActin and values are expressed as mean fold changes $( \pm \mathrm{SD})$ relative to the transcript level of AtLecRK-I.9 in I.9-OE-4 or the transcript level of AtLecRK-IX.1 in IX.1-OE-8 that was arbitrarily set as 1. d GFP, LecRK-I.9-eGFP and LecRK-IX.1-eGFP accumulation in transgenic $N$. benthamiana lines. Proteins were immunodetected using antiGFP-HRP. Coomassie staining was used to indicate the amount of loading in each lane

with two or more copies. For example, line I.9-OE-16 contains a single AtLecRK-I.9 copy but showed the highest transgene expression level of all I.9-OE lines. Even transgenic lines with the same transgene number, e.g. line IX.1OE-3 and IX.1-OE-11, showed quite different expression levels (Fig. 2c). To determine whether the transgenic lines produce the LecRK proteins, we isolated proteins and performed western blot analysis using GFP antibody. For this analysis we selected a subset of seven I.9-OE lines and three IX.1-OE lines that varied in transgene copy number and expression. In two EV lines (i.e. EV-2 and EV-4), different amounts of GFP with the expected size around $28 \mathrm{kDa}$ were detected. In the I.9-OE lines, variable amounts of LecRK-I.9-eGFP were detected (Fig. 2d) and comparison with the expression levels (Fig. 2c) suggests that the accumulation of the LecRK-I.9 is correlated with the transgene expression level. LecRK-IX.1-eGFP was only detected in one of the three IX.1-OE lines, and this is the one, IX.1-OE-10, with the highest transgene expression level of the three selected IX.1-OE lines (Fig. 2d).

\section{Morphology and growth alterations of I.9-OE and IX.1-OE lines}

In a previous study, it was reported that overexpression of AtLecRK-I.9 in Arabidopsis led to more compact rosettes with smaller and slightly wrinkled leaves (Bouwmeester et al. 2011). In line with this, ectopic expression of AtLecRK-I.9 in potato also led to developmental defects, such as aberrant leaf morphology (Bouwmeester et al. 2014). In this study, the $\mathrm{T}_{0}$ transgenic $N$. benthamiana plants were monitored for growth and developmental alterations starting from 1-week after transfer into soil until seed set (Table 2; Fig. 1). All the four EV lines showed normal development; i.e. leaf morphology, branching and plant height were similar to untransformed $N$. benthamiana. In contrast, several I.9-OE lines were smaller in size and displayed more compact rosettes or curly leaves. These phenotypic changes, however, were not correlated with AtLecRK-I.9 expression levels, but this could be due to a combined effect of varying transgene copy numbers, transgene insertion sites and transgene expression levels in the different transgenic lines. Three out of the twelve IX.1OE lines, namely IX.1-OE-10, IX.1-OE-11 and IX.1-OE12 , showed spontaneous cell death in leaves of over 5-week-old plants (Fig. 3a), a phenomenon that was not observed in any of the $N$. benthamiana lines expressing LecRK-I.9. Considering that these three IX.1-OE lines showed much higher AtLecRK-IX.1 expression levels than the rest, the cell death phenotype might be attributed to the elevated AtLecRK-IX.1 expression. This is supported by our previous observations reported in Wang et al. (2015b) that transient expression of AtLecRK-IX.1 in N. benthami$a n a$ also enhances cell death. Moreover, a similar cell death phenotype was found when AtLecRK-IX.1 was overexpressed in Arabidopsis, and this was also shown to be correlated with AtLecRK-IX.1 expression levels (Wang et al. 2015b).

To determine whether the observed LecRK-mediated phenotypes are maintained in the offspring, two EV lines, nine I.9-OE lines and eight IX.1-OE lines were propagated and their segregating progeny ( $\mathrm{T}_{1}$ plants) was assayed for transgene presence and alteration in morphology and development of spontaneous cell death. As indicated in Table 2, the phenotypes in $T_{1}$ plants containing the transgene were similar to those observed in $\mathrm{T}_{0}$ lines. Spontaneous cell death was only found in progeny of IX.1-OE lines that has high transgene expression (Fig. 3b; Table 2). These plants also showed retarded growth when compared to those without AtLecRK-IX.1 (Fig. 3c) or with low transgene expression (Table 2). Based on these observations, we conclude that the observed morphological and cell death phenotypes in these lines are due to the presence of the AtLecRK-IX.1 transgene and not to a random gene insertion effect. In a previous study, we showed that mutation of the catalytic RD-motif within LecRK-IX.1 abolishes induction of cell death and pathogen resistance (Wang et al. 2015b). Hence, we anticipate that the induced cell death is either directly mediated by LecRK-IX or indirectly through constitutive activation of defense.

For all the EV and IX.1-OE lines, seeds harvested from $\mathrm{T}_{0}$ plants have a similar germination efficiency as untransformed $N$. benthamiana when grown in soil or on MS medium (Table 2; Fig. 3d). However, 10 out of the 16 I.9OE lines showed severe defects in seed germination, with a germination rate of $60 \%$ or lower (Table 2). Also here, the 
a

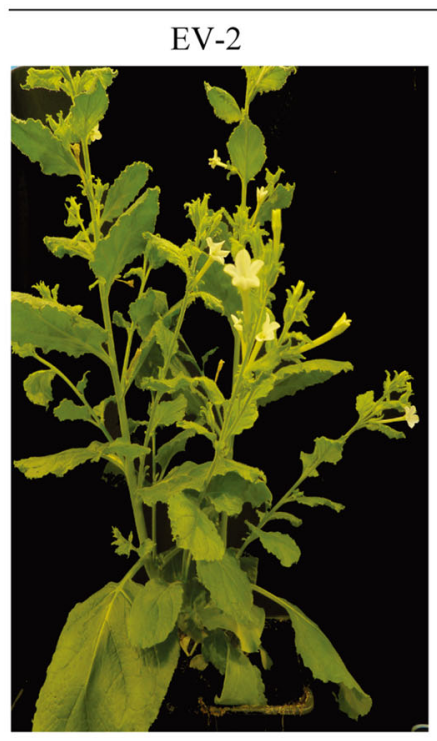

$\mathrm{T} 0$

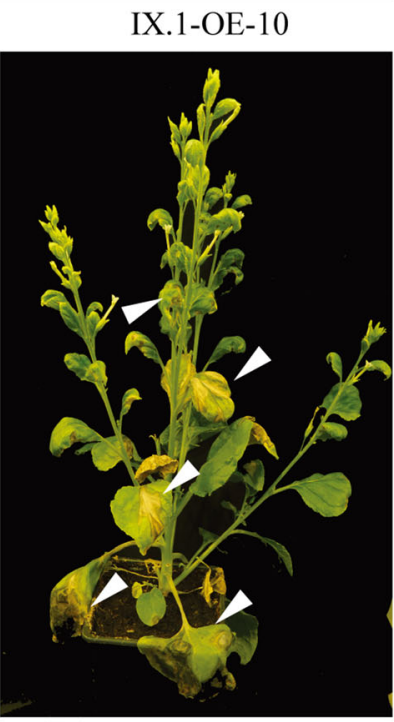

b

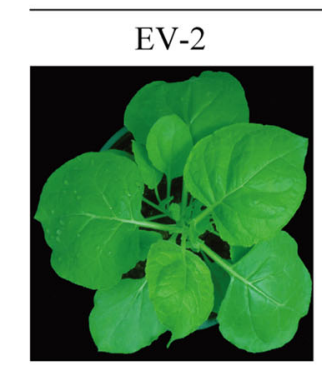

c

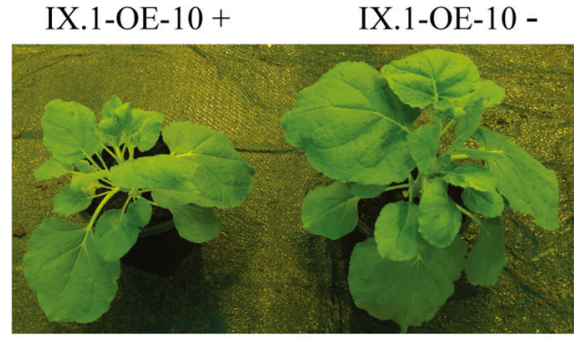

I.9-OE-17

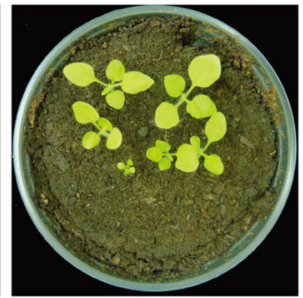

$\mathrm{T} 1$

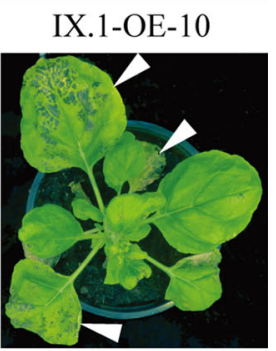

IX.1-OE-10

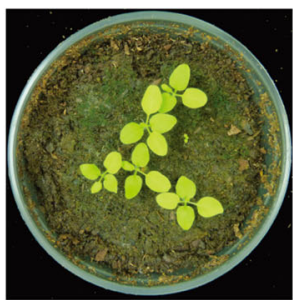

Fig. 3 Morphology of transgenic $N$. benthamiana lines. a The $\mathrm{T}_{0}$ $N$. benthamiana IX.1-OE-10 plant, but not the $\mathrm{T}_{0}$ EV-2 plant displayed cell death. Ten-week-old plants were photographed. b The $\mathrm{T}_{1} N$. benthamiana IX.1-OE-10 plant, but not the EV-2 plant developed cell death. Six-week-old plants were photographed. c $\mathrm{T}_{1}$ progeny of the IX.1-OE-10 line harboring AtLecRK-IX.1 (+) is

severity of the phenotype does not correlate with AtLecRKI.9 expression levels in the various transgenic lines.

\section{I.9-OE and IX.1-OE lines show enhanced resistance to Phytophthora pathogens}

Both LecRK-I.9 and LecRK-IX.1 were previously shown to function in Phytophthora resistance in Arabidopsis (Bouwmeester et al. 2011; Wang et al. 2015b). In order to determine whether both LecRKs retain their function in Phytophthora resistance, we checked the response of N. benthamiana I.9-OE and IX.1-OE lines upon inoculation with $P$. capsici and $P$. infestans. Leaves from $\mathrm{T}_{1}$ progenies harboring the transgenes were used for infection assays. It has to be noted that for lines with a cell death phenotype only leaves without any visible cell death symptoms were used for infection assays. Upon pluginoculation with P. capsici LT263 or LT3239, smaller lesions were found on the I.9-OE and IX.1-OE lines when smaller in size than the $\mathrm{T}_{1}$ progeny without AtLecRK-IX.1 (-). Sixweek-old plants were photographed. d Germination of seeds harvested from untransformed and transgenic $N$. benthamiana plants. Six seeds were sown in each pot. Two-week-old seedlings were photographed

compared to EV-1 and EV-2 (Fig. 4a, b), indicating that constitutive expression of AtLecRK-I.9 or AtLecRK-IX.1 in $N$. benthamiana enhances resistance to different isolates of $P$. capsici. This increased resistance was also found in I.9OE and IX.1-OE lines when inoculated with $P$. infestans (Fig. 4c, d). As shown in Fig. 4e, all tested transgenic lines showed reduced lesion sizes when compared with those on EV control plants. There was however, no indication for a correlation between lesion size and the level of transgene expression (Fig. 2; Table 2). For example, lesion sizes on lines with high transgene expression levels (e.g. I.9-OE-10 and I.9-OE-17) were found to be comparable with those displayed on lines that have low transgene expression levels (e.g. I.9-OE-9). On the other hand lines that have one transgene copy with similar transgene expression levels were found to vary significantly in lesions sizes (e.g. I.9OE-5 and I.9-OE-7). These findings show that the resistance phenotype in these transgenic lines is regulated at different levels and cannot be entirely attributed to the 
Fig. 4 Infection assays on transgenic $N$. benthamiana lines with different Phytophthora pathogens. a-c Disease symptoms on transgenic $N$. benthamiana EV, I.9-OE and IX.1-OE lines 3 days after pluginoculation with $P$. capsici LT263 (a) or LT3239 (b), or 6 days after zoosporeinoculation with $P$. infestans 14-3-GFP (c). Lesions are indicated by black circles. d Average lesion sizes on N. benthamiana plants upon inoculation with Phytophthora pathogens. Each experiment included 12-20 inoculation sites. Bars represent the mean lesion sizes $( \pm \mathrm{SE})$. Asterisks indicate significant difference in lesion sizes $(p<0.01)$ compared to the EV lines based on One-way ANOVA with Tukey's HSD test. Infection assays were repeated three times with both $P$. capsici isolates and twice with $P$. infestans with similar results. e Average lesion sizes on $N$. benthamiana plants inoculated with $P$. infestans. Each experiment included 12-20 inoculation sites. Bars represent the mean lesion sizes $( \pm \mathrm{SE})$. Asterisks indicate significant difference in lesion sizes $(p<0.01)$ compared to the EV lines based on One-way ANOVA with Tukey's HSD test. Infection assays were repeated twice with similar results $\mathbf{a}$ P. capsici LT263

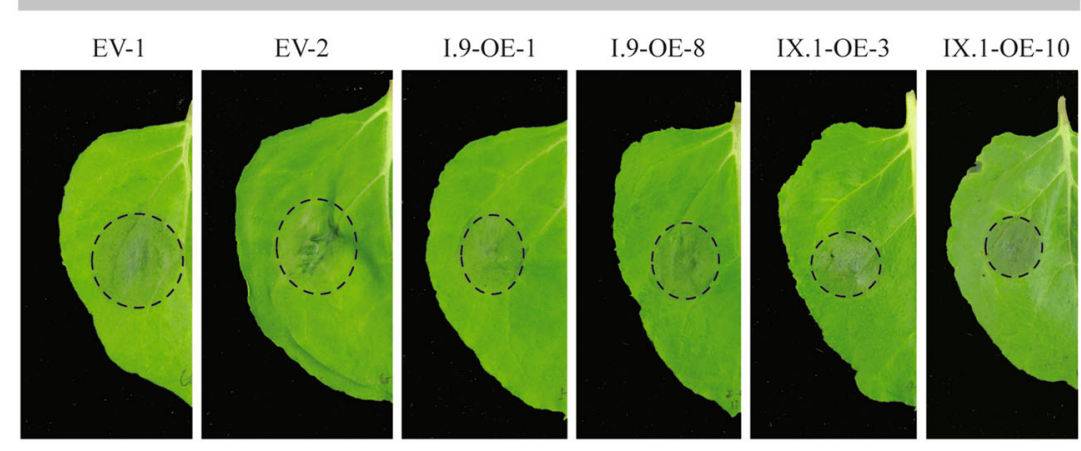

b

P. capsici LT3239
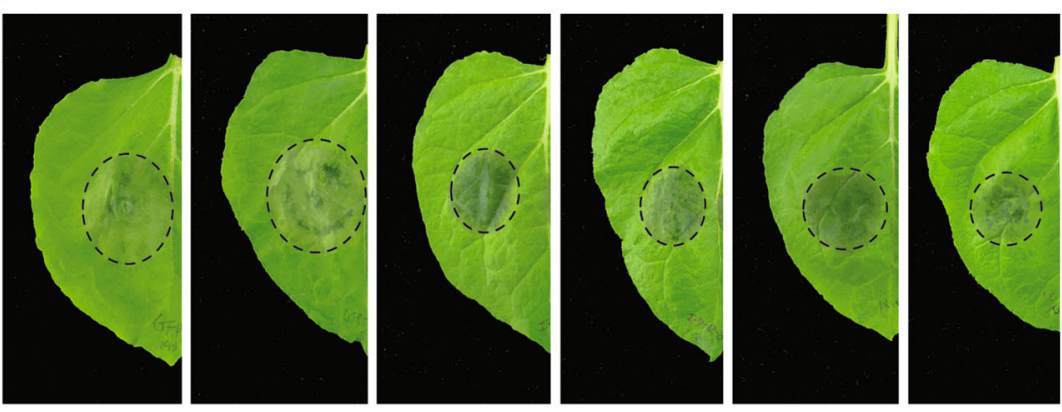

c

P. infestans 14-3-GFP
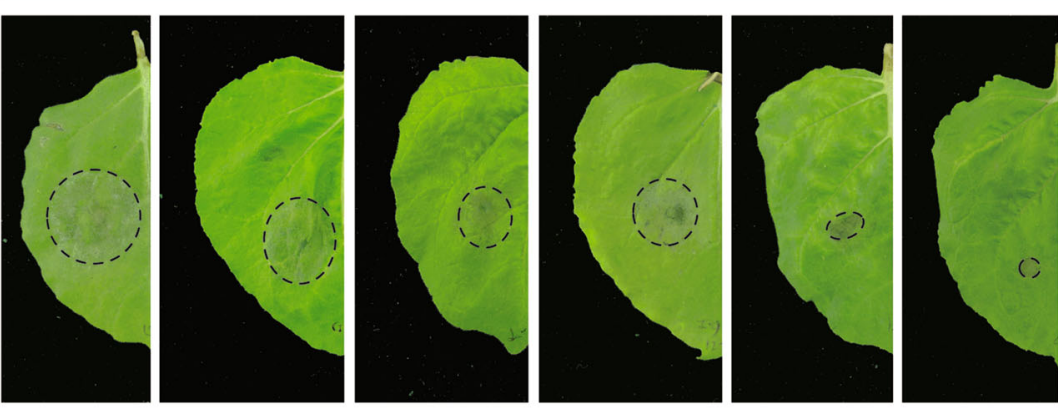

d
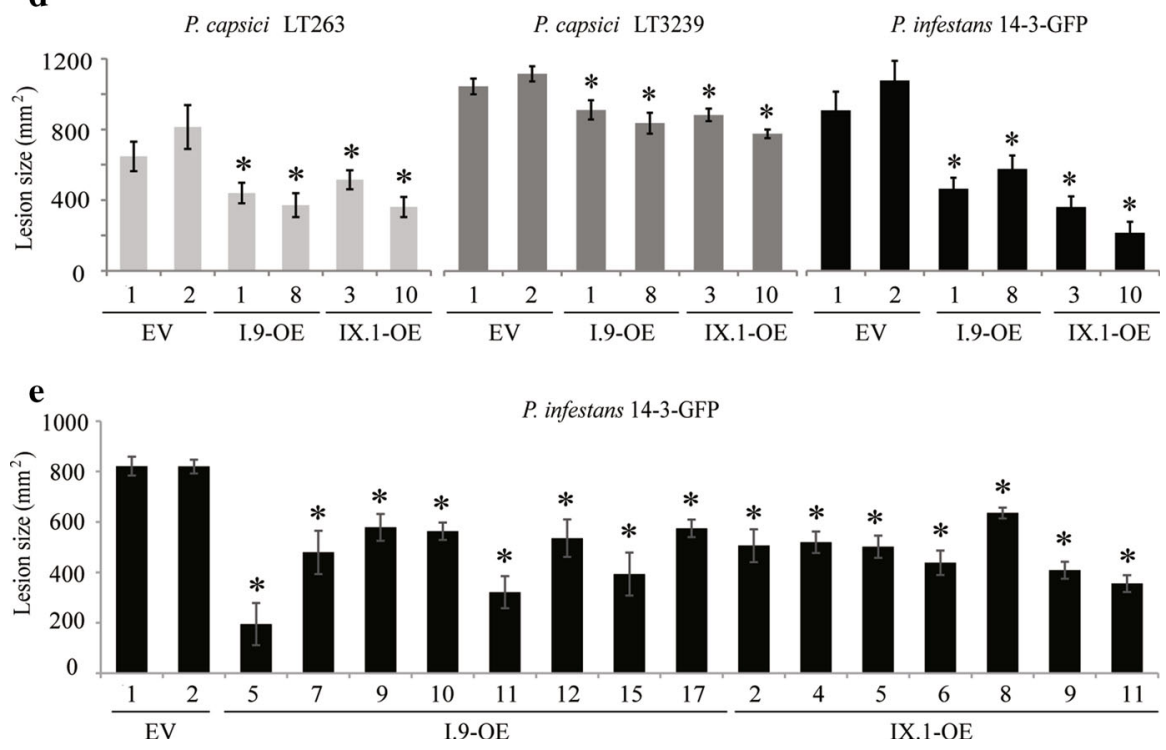
level of transgene expression or the transgene copy number.

\section{Conclusions}

In this study, multiple transgenic $N$. benthamiana lines with constitutive expression of Arabidopsis LecRK-I.9 or LecRK-IX.1 were obtained. Transgenic lines varied in transgene copy number, transgene expression level and protein accumulation. Ectopic expression of either AtLecRK-I.9 or AtLecRK-IX.1 in N. benthamiana increased the resistance to different Phytophthora species. Our results suggest that Arabidopsis LecRK-I.9 and LecRK-IX.1 maintained their function in Phytophthora resistance when transferred into $N$. benthamiana, which is in line with results that we obtained previously with transgenic potato plants expressing LecRK-I.9 (Bouwmeester et al. 2014). These findings suggest that LecRKs could be used as complementary resistance components, in combination with canonical NLR-encoding $R$ genes, for engineering broad-spectrum disease resistance to Phytophthora pathogens. However, ectopic expression of both LecRKs also caused several adverse effects on plant fitness, such as curly leaves, leaf necrosis or reduced plant size. In the case of LecRK-IX.1, its function in Phytophthora disease resistance is independent of that in plant cell death induction (Wang et al. 2015b). Therefore, we anticipate that it should be possible to optimize the receptors in such a way that downstream signaling does no longer cause plant growth alterations while the LecRK-mediated disease resistance is maintained. The transgenic $N$. benthamiana lines that we describe in this study can be used as a valuable experimental tool for further analysis of the components required for LecRK-mediated resistance and plant growth alterations, for example via the virus-induced gene silencing or protein complex pull-down assays.

Author contribution statement Y.W., F.G. and K.B. conceived and designed research. Y.W., D.L.N. and H.M.J. carried out the experiments and analysed data. Y.W., F.G. and K.B. wrote the manuscript. All authors read and approved the manuscript.

Acknowledgments We thank Bert Essenstam from Unifarm for plant care. This project was financially supported by a Wageningen University sandwich-PhD fellowship (Y.W.), a Huygens scholarship (Y.W.), the Netherlands Fellowship Program (D.L.N. and H.M.J.), a VENI grant (K.B.) from the Netherlands Organization for Scientific Research (Technology Foundation NWO-STW), and the Food-forThought campaign of the Wageningen University fund.

\section{Compliance with ethical standards}

Conflict of interest The authors declare that they have no conflict of interest.
Open Access This article is distributed under the terms of the Creative Commons Attribution 4.0 International License (http://crea tivecommons.org/licenses/by/4.0/), which permits unrestricted use, distribution, and reproduction in any medium, provided you give appropriate credit to the original author(s) and the source, provide a link to the Creative Commons license, and indicate if changes were made.

\section{References}

Bouwmeester K, Govers F (2009) Arabidopsis L-type lectin receptor kinases: phylogeny, classification, and expression profiles. J Exp Bot 60:4383-4396

Bouwmeester K, de Sain M, Weide R, Gouget A, Klamer S, Canut H, Govers F (2011) The lectin receptor kinase LecRK-I.9 is a novel Phytophthora resistance component and a potential host target for a RXLR effector. PLoS Pathog 7:e1001327

Bouwmeester K, Han M, Blanco-Portales R, Song W, Weide R, Guo L, van der Vossen EAG, Govers F (2014) The Arabidopsis lectin receptor kinase LecRK-I.9 enhances resistance to Phytophthora infestans in Solanaceous plants. Plant Biotechnol J 12:10-16

Fry W (2008) Phytophthora infestans: the plant (and $R$ gene) destroyer. Mol Plant Pathol 9:385-402

Hofberger JA, Nsibo DL, Govers F, Bouwmeester K, Schranz ME (2015) A complex interplay of tandem- and whole genome duplication drives expansion of the L-type lectin receptor kinase gene family in the Brassicaceae. Genome Biol Evol 7:720-734

Honda M, Muramoto Y, Kuzuguchi T, Sawano S, Machida M, Koyama H (2002) Determination of gene copy number and genotype of transgenic Arabidopsis thaliana by competitive PCR. J Exp Bot 53:1515-1520

Huang PY, Yeh YH, Liu AC, Chien CP, Zimmerli L (2014) The Arabidopsis LecRK-VI.2 associates with the pattern-recognition receptor FLS2 and primes Nicotiana benthamiana patterntriggered immunity. Plant J 79:243-255

Kole C, Michler C, Abbott A, Hall T, Kohli A, Miro B, Twyman R (2010) Transgene integration, expression and stability in plants: strategies for improvements. In Transgenic crop plants. Springer, Berlin, pp 201-237

Kunze G, Zipfel C, Robatzek S, Niehaus K, Boller T, Felix G (2004) The $\mathrm{N}$ terminus of bacterial elongation factor Tu elicits innate immunity in Arabidopsis plants. Plant Cell 16:3496-3507

Lacombe S, Rougon-Cardoso A, Sherwood E, Peeters N, Dahlbeck D, van Esse HP, Smoker M, Rallapalli G, Thomma BPHJ, Staskawicz BJ, Jones JDG, Zipfel C (2010) Interfamily transfer of a plant pattern-recognition receptor confers broad-spectrum bacterial resistance. Nat Biotechnol 28:365-369

Lamour KH, Stam R, Jupe J, Huitema E (2012) The oomycete broadhost-range pathogen Phytophthora capsici. Mol Plant Pathol 13:329-430

Rodewald J, Trognitz B (2013) Solanum resistance genes against Phytophthora infestans and their corresponding avirulence genes. Mol Plant Pathol 14:740-757

Tyler BM (2007) Phytophthora sojae: root rot pathogen of soybean and model oomycete. Mol Plant Pathol 8:1-8

Vleeshouwers VGAA, van Dooijeweert W, Keizer LCP, Sijpkes L, Govers F, Colon LT (1999) A laboratory assay for Phytophthora infestans resistance in various Solanum species reflects the field situation. Eur J Plant Pathol 10:241-250

Vleeshouwers VGAA, Raffaele S, Vossen JH, Champouret N, Oliva R, Segretin ME, Rietman H, Cano LM, Lokossou A, Kessel G, Pel MA, Kamoun S (2011) Understanding and exploiting late blight resistance in the age of effectors. Annu Rev Phytopathol 49:507-531 
Wang Y, Bouwmeester K, van de Mortel JE, Shan W, Govers F (2013) A novel Arabidopsis-oomycete pathosystem: differential interactions with Phytophthora capsici reveal a role for camalexin, indole glucosinolates and salicylic acid in defence. Plant Cell Environ 36:1192-1203

Wang Y, Bouwmeester K, Beseh P, Shan W, Govers F (2014) Phenotypic analyses of Arabidopsis T-DNA insertion lines and expression profiling reveal that multiple L-type lectin receptor kinases are involved in plant immunity. Mol Plant-Microbe Interact 27:1390-1402

Wang Y, Weide R, Govers F, Bouwmeester K (2015a) L-type lectin receptor kinases in Nicotiana benthamiana and tomato and their role in Phytophthora resistance. J Exp Bot. 66:6731-6743
Wang Y, Cordewener JHG, America AHP, Shan W, Bouwmeester K, Govers F (2015b) Arabidopsis lectin receptor kinases LecRKIX.1 and LecRK-IX.2 are functional analogs in regulating Phytophthora resistance and plant cell death. Mol Plant-Microbe Interact 28:1032-1048

Zipfel C (2014) Plant pattern-recognition receptors. Trends Immunol 35:345-351

Zipfel C, Kunze G, Chinchilla D, Caniard A, Jones JDG, Boller T, Felix G (2006) Perception of the bacterial PAMP EF-Tu by the receptor EFR restricts Agrobacterium-mediated transformation. Cell 125:749-760 